\title{
Challenges and trends in wireless ubiquitous computing systems
}

\author{
Abdelfettah Belghith • Anis Koubaa • \\ Elhadi Shakshuki
}

Published online: 10 April 2011

(C) Springer-Verlag London Limited 2011

In the last decade, the Internet paradigm has been evolving toward a new frontier with the emergence of ubiquitous and pervasive systems, including wireless sensor networks, ad hoc networks, RFID systems, and wireless embedded systems. In fact, while the initial purpose of the Internet was to interconnect computers to share digital data at large scale, the current tendency is to enable ubiquitous and pervasive computing to control everything anytime and at a large scale. This new paradigm has given rise to a new generation of networked systems, commonly known as Internet-of-Things or Cyber-Physical Systems.

The research community has actively investigated the underlying challenges pertaining to these systems, as they fundamentally differ from the classical problems due to their inherent constraints. This special issue presents six papers covering various topics in personal and ubiquitous computing.

The first paper entitled "RiSeG: A Ring Based Secure Group Communication Protocol for Wireless Sensor Networks" deals with security in ubiquitous systems. The paper presents a new approach for secure group management in wireless sensor networks. The proposed approach

\footnotetext{
A. Belghith $(\square)$

University of Manouba, Manouba, Tunisia

e-mail: abdelfattah.belghith@ensi.rnu.tn
}

\author{
A. Koubaa \\ e-mail: aska@isep.ipp.pt \\ A. Koubaa \\ CISTER Research Unit, Porto, Portugal \\ E. Shakshuki \\ Acadia University, Wolfville, Canada \\ e-mail: elhadi.shakshuki@acadiau.ca
}

Al-Imam Mohamed bin Saud University, Riyadh, Saudi Arabia is based on a logical ring architecture, which permits to alleviate the group controller's task in updating the group key. The proposed scheme also provides backward and forward secrecy, addresses the node compromise attack, and gives a solution to detect and eliminate the compromised nodes. The authors evaluated their scheme in terms of storage, computation, and communication costs and compared its behavior against the Logical Key Hierarchy (LKH) scheme. It has been shown that RiSeG requires less storage cost and reduces computation and communication costs at the Group Controller as compared with LKH. The paper goes beyond theoretical work and proposes a realworld implementation, which first proved that RiSeG is applicable to WSNs and also showed that the performance results in terms of execution time, energy consumption, and memory consumption are satisfactory.

The second paper entitled "Stability routing with constrained path length for improved routability in dynamic MANETs" addresses the problem of enhancing the routing validity in mobile wireless multi hop ad hoc networks. In proactive routing, routes are established and updated periodically and consequently loose their pertinence as time tics away from the start of their updating instants. Authors argue that routability, defined as the validity of established routes, stands among the most centric metric impacting the network efficiency. Indeed, in dynamic networks, traffic routed through invalid routes not only amounts to a waste of valuable resources but will never be delivered to its destination. In the quest of improving routability in dynamic networks, the authors considered a two-constrained QoS routing problem with one superlative constraint and one comparative constraint. In its general form, this is an NP hard problem. The authors proposed a novel exact algorithm and then instantiated the problem to solve the optimal integer weight-constrained path length 
problem in polynomial time. Furthermore, they set up a common framework to compare three families of proactive routing: routing based on shortest path, routing based on the most stable path, and the proposed routing based on the most stable constrained path. They showed that an adequate compromise between route stability and its length in hops is essential for mitigating the impact of the routing dynamics. Extensive simulation results exacerbated the appropriateness and the efficiency of their proposed most stable constrained path routing algorithm $(O C n P P)$ in terms of a high routability and a low average path length.

The third paper entitled "Robust Video Communication for Ubiquitous Network Access" addresses the application of a window-growth rateless code for data-partitioned video to provide good video quality at a small decoding delay. Data partitioning (DP) prioritizes the more important data within a compressed bitstream. The paper demonstrates an innovation in the application of window-growth rateless coding for video data partitioning, which is able to lessen transmission delay with reduced redundant overhead. The authors provided details for achieving this scheme in the context of an H264/AVC codec's picture types and structures. They conducted simulations to model both wireless and wired access networks. They showed that their unequal protection proposed scheme achieves a video quality improvement of several dBs when compared with equal protection.

The fourth paper entitled "A New Model for Contextaware Transactions in Mobile Services" concerned with the absence of current transaction models and commit protocols that do not take into account context information. The authors of this paper proposed a new model for context-aware transactions and their performance management in mobile environments. The authors also presented a protocol, which is used to execute the context-aware transactions in mobile services. The protocol ensures that context-aware transactions enforce the RACCD criteria that are crucial to maintaining the correctness of M-services and the consistency of their underlying data. A prototype system is developed and demonstrated to test the validity of the proposed model. A number of performance management experiments have been conducted to evaluate the performance of context-aware transactions in terms of processing time, message delay, and transaction throughput.
The fifth paper entitled "A New Scalable Multicast Routing Algorithm for Interactive Real-Time Applications" deals with the support of real-time multicast communications where not only the end-to-end delay is constrained to a certain bound (known as the multicast endto-end delay problem), but also the delay variation on the different paths should be kept within a definite limit (known as the delay-variation problem). They proposed a new and novel strategy for constructing multi-constrained multicast trees. Firstly, the authors derived mathematically a new delay-variation estimation scheme and proved its efficiency. Secondly and based on the proposed estimation scheme, they proposed a simple and efficient heuristic algorithm to jointly solve the delay and delay variationconstrained routing problem. Through extensive simulations, they showed that their proposed QoS routing algorithm $(C D D V)$ outperforms the well known (DVDMR) in terms of multicast delay variation.

The sixth paper entitled "Optimized Skyline Queries on Road Networks using Nearest Neighbors" tackles the spatial network skyline query's problem that needs the calculation of attributes with an expensive distance calculation operation. Toward this end, the authors proposed an efficient algorithm that requires a remarkably less number of network distance calculations between the query points and data points. Their approach uses a progressive nearest neighbor algorithm to minimize the set of candidates and then evaluates those candidates by only comparing them with a subset of discovered skyline points. The algorithm operates on three main phases: nearest neighbor computation, distance computation, and dominance tests. Performing first and second phases resulted in minimizing the number of distance calculation operations to $O(n q)$, where $n q$ is the number of query points. Authors performed some experiments to demonstrate the effectiveness of the proposed algorithm as to previous works.

The guest co-editors would like to take this opportunity to thank the authors for the efforts they put in the preparation of their manuscripts and for their valuable contributions. We wish to express our deepest gratitude to the referees for their thorough detailed reviews. Last, but not least, our sincere thanks go to the Editor-in-Chief Dr. Peter Thomas of the Journal of Pervasive and Ubiquitous Computing for the exceptional support and assistance he provided throughout this process. 